\title{
Efecto híbrido en la demanda del profesional publicitario: un reto ante la transformación digital en la publicidad
}

\section{Erika P. Álvarez-Flores ${ }^{1}$ Patricia Núñez Gómez² Luis Mañas Viniegra ${ }^{3}$}

Recibido: 15/01/2020

Aprobado por pares: 13/03/2020
Enviado a pares: $15 / 01 / 2020$

Aceptado: 13/03/2020

DOI: 10.5294/pacla.2021.24.2.4

Para citar este artículo / to reference this article / para citar este artigo

Álvarez-Flores, E. P., Núñez, P. y Mañas, L. (2021). Efecto híbrido en la demanda del profesional publicitario: un reto ante la transformación digital en la publicidad. Palabra Clave, 24(2), e2424. DOI: https://doi.org/10.5294/pacla.2021.24.2.4

\section{Resumen}

La rápida evolución de la comunicación y de la interrelación social afecta la situación sociolaboral y hace que los escenarios del mercado profesional se modifiquen constantemente. La convergencia de los medios de comunicación e internet ha creado otras condiciones para la transmisión de información y mensajes que inciden en la industria publicitaria. Esta transformación del ecosistema digital, donde el consumidor interactúa a través de varios medios y canales, plantea una modificación de las demandas de empleo que conlleva la interrelación entre perfiles. A través de una revisión de la literatura existente, el análisis de ofertas de trabajo y el contraste 
con entrevistas a nueve expertos en Comunicación Comercial, se explora el efecto de hibridación de perfiles profesionales para graduados en Publicidad, ante los requerimientos de la industria de la comunicación comercial en España. El diagnóstico posibilita un mayor entendimiento de los perfiles híbridos en auge en el ámbito laboral publicitario, cuyo fin es mejorar la experiencia del consumidor ante el marcado vínculo con lo digital.

\section{Palabras clave (Fuente: tesauro de la Unesco)}

Competencia profesional; digitalización; estatus profesional; mercado de trabajo; publicidad. 


\section{Hybrid Effect on the Demand for Advertising Executives: A Challenge in the Face of the Digital Transformation of Advertising}

\section{Abstract}

The rapid evolution of communication and social interrelation affects the socio-labor situation and causes the professional market scenarios to change constantly. The convergence of the media and the internet has created other conditions to convey information and messages that impact the advertising industry. This transformation of the digital ecosystem, where the consumer interacts through various media and channels, modifies job demands and entails profile interrelation. Through a review of the existing literature, the analysis of job offers, and the corroboration with interviews with nine Business Communication experts, we explore the effect of hybridization of professional profiles on Advertising graduates in light of the requirements of the business communication industry in Spain. The diagnosis provides a better understanding of the hybrid profiles on the rise in the advertising labor environment, whose purpose is to improve the consumer experience regarding its strong link with the digital world.

\section{Keywords (Source: Unesco Thesaurus)}

Occupational qualifications; digitization; professional status; labour market; advertising. 


\section{Efeito híbrido na demanda do profissional publicitário: um desafio ante a transformação digital na publicidade}

\section{Resumo}

A rápida evolução da comunicação e da inter-relação social afeta a situação sociolaboral e faz com que os cenários do mercado profissional sejam modificados constantemente. A convergência entre os meios de comunicação e a internet vem criando condições para a transmissão de informações e mensagens que impactam na indústria publicitária. Essa transformação do ecossistema digital, em que o consumidor interage por meio de vários meios e canais, propõe uma modificação das demandas de emprego que implica a inter-relação entre perfis. A partir de uma revisão da literatura, da análise de ofertas de trabalho e do contraste com entrevistas a nove especialistas em comunicação comercial, o efeito de hibridização de perfis profissionais para formados em Publicidade é explorado, ante as exigências da indústria da comunicação comercial na Espanha. Os resultados possibilitam maior entendimento dos perfis híbridos que estão no auge no contexto do trabalho publicitário, cujo objetivo é melhorar a experiência do consumidor diante do marcado vínculo com o digital.

\section{Plavras-chave (Fonte: tesauro da Unesco)}

Competência profissional; digitalização; status profissional; mercado de trabalho; publicidade. 
La digitalización produjo una multiplicidad de medios y canales que cambiaron profundamente los hábitos y usos de la sociedad en el modo de comprar, entretenernos y relacionarnos. Ante este hecho, y para asumir esos retos, era inevitable la transición en la comunicación publicitaria (Kumary Gupta, 2016; Bravo y Madinaveitia, 2015). Además, se ha constatado que la publicidad online es ahora un elemento clave del ecosistema digital (Ontsi, 2017) para comunicar a una sociedad hiperconectada (Perlado, Saavedra y Rubio, 2016), con acceso ilimitado a servicios de internet. Este impulso tecnológico enfrenta a las empresas a desarrollar cambios estructurales, debido a la demanda de profesionales formados en competencias digitales que requiere la comunicación comercial, ante otros tipos de audiencias en internet, como se detalla en el informe PAPETVII (Rooter, 2012) y en el informe del Observatorio del Mercado Laboral de profesionales de la comunicación y la publicidad (IAB, 2011).

Los cambios digitales han traído consigo una evolución que transforma la publicidad en sí y produce una reordenación de perfiles profesionales para publicidad, donde se atenúan los criterios online y offline para trabajar en ambientes más integrados (De-Salas, Martínez y Roca, 2019). El mercado publicitario y la propia publicidad continúan en transición con las iniciativas económicas y sus exigencias (Observatorio de la Publicidad en España, 2015). La consolidación de un ecosistema de medios eminentemente digital ya propició que en los perfiles clásicos de la comunicación sean necesarias competencias que contemplen requerimientos tecnológicos (Armendáriz, 2015; Besalú, Schena y Sánchez, 2017; Sánchez y Fernández, 2018) y que, dependiendo de la intensidad de involucramiento con dichos conocimientos técnicos, se determine el nivel profesional de los sujetos (Fuller et al., 2018). Ante la importancia que cobra la comunicación para el mercado publicitario en medios sociales y/o el posicionamiento en la web, se han destacado cambios en las estructuras laborales, para hacerlo de la forma más eficiente posible, aumentando las funciones vinculadas (Álvarez, Núñez y Olivares, 2018; Schultz, 2016) o la complejidad en los procesos de trabajo (Perlado, Papí y Bergaz, 2019). En consecuencia, se ha estimulado el reciclaje de profesionales para dar respuesta a las necesidades que trae el despliegue tecnológico en la actividad publicitaria. 
En este sentido, Perlado (2013) constata la inclinación del mercado laboral a interrelacionar los conocimientos técnicos con las habilidades tradicionales de la comunicación, y delimita la necesidad de figuras con funciones que integren varios perfiles. Otros perciben que las empresas buscan un profesional versátil o "todo terreno" (López, Papí y Martín, 2016; Sánchez y Fernández, 2018), y se confirma una polivalencia laboral marcadamente digitalizada en el área de comunicación (Ventura, Roca y Corral, 2018). En tal sentido, Toledano, Miguel y Grijalba (2017) y Álvarez et al. (2018) observan que el comportamiento del consumidor, que cada día es más digital y enfocado a elementos online, hace que sean comunes en las empresas publicitarias perfiles profesionales multidisciplinarios ligados a internet, posicionamiento web, community manager, Search Engine Optimitation (SEO) y Search Engine Marketing (SEM). Se remarca la exigencia en el mercado laboral de conocimientos específicos del área, pero asociados al campo de los contenidos digitales, en lo que toca tanto a los conocimientos informáticos y de programación como a las capacidades creativas y artísticas, para satisfacer de la manera más eficiente y creativa el aumento de audiencias en medios sociales y comunidades digitales, que juegan un papel relevante en la comunicación.

A la luz del avance digital, se exploró en primer término la información que proporciona la investigación de perfiles donde se demanda profesionales de la publicidad formados en competencias digitales. Con ello, este estudio pretende contrastar, en la realidad del sector y a través de ofertas de trabajo y profesionales publicitarios, el efecto de hibridación de perfiles para graduados en Publicidad, hibridación que se ha dado en el ámbito laboral de la actividad publicitaria a raíz del marcado vínculo con lo digital, que ha llevado a las empresas a establecer espacios virtuales de interacción con el consumidor.

\section{Metodología}

Con el objetivo de comprender cómo lo digital está modificando los perfiles de la actividad publicitaria en un sentido más híbrido, se utilizó una técnica mixta basada en el análisis de contenido y el análisis del discurso. 
Primeramente, se exploraron estudios sobre demandas profesionales para graduados en Publicidad surgidos tras la digitalización en la comunicación comercial en España y se los delineó con base en el Libro blanco: títulos de grado en comunicación para el diseño de las titulaciones en la economía digital (MIET, 2015). Para reflexionar sobre la realidad de perfiles híbridos en los actuales roles laborales, se examinaron ofertas de trabajo y sus competencias asociadas en un portal relevante para el área de comunicación publicitaria en el territorio de España. Además, se reforzaron los resultados extraídos de la revisión, con entrevistas en profundidad a profesionales del sector publicitario, donde se recogió su percepción sobre la realidad de perfiles híbridos -que podrían diferir con respecto a las vacantes que se ocupan mediante la publicación de ofertas, en vez de recurrir a las redes de contactos-, así como sobre los que están en auge.

\section{Instrumentos}

Para el análisis de ofertas de trabajo se consideraron todos los anuncios publicados en el portal Domestika ( $h$ ttps://www.domestika.org/es/jobs) en períodos diferenciados: el primero de ellos en los meses de febrero a abril de 2018 y el segundo de febrero a abril de 2019. Se emplearon múltiples períodos para garantizar la validez de los resultados y la obtención de mayores dosis de fiabilidad, precisión y objetividad, al observar si existe variabilidad a la hora de solicitar perfiles híbridos por las empresas posterior a las entrevistas a expertos. Para refrendar los resultados, además, se observaron ofertas de diciembre de 2019. Los anuncios en los que se profundizó fueron extraídos de un muestreo estratificado en los que debían aparecer términos relacionados con las palabras clave "profesionales de publicidad" y "publicidad digital" en la descripción interna de la oferta, y se desestimaron aquellos que no estaban orientados a ellos. En cada período, se encontraron más de 40 ofertas vinculadas al perfil. La información de estos anuncios fue vaciada en una ficha de recogida de datos que contenía variables de identificación y características claves orientadas a competencias tecnológicas y destrezas no tecnológicas.

Entre abril y julio de 2018, se realizaron entrevistas semiestructuradas a 9 expertos, triangulados por grupos, orientadas a detectar la presen- 
cia híbrida de perfiles, de manera que la muestra ofreciera diferentes puntos de vista (Malhotra et al., 2002), desde la triple perspectiva de la agencia, el anunciante y los medios. A partir del universo de agencias, anunciantes y medios en España, se empleó una muestra incidental no probabilística formada por una selección de profesionales descrita en la Tabla 1 y cuya confidencialidad se mantiene más allá de su perfil como expertos, ya que algunos participantes no autorizaron su identificación en el estudio. Las entrevistas en profundidad semiestructuradas constituyen un método utilizado habitualmente en investigaciones exploratorias en el ámbito de la comunicación (Kvale, 2011; McEnnis, 2017). Es un método cualitativo indicado para entrevistados poco accesibles por su responsabilidad profesional y por tratarse de temas comprometidos con su actividad profesional (Vilches, 2011), cuestión que dificulta la participación de estos.

\section{Tabla 1. Perfil profesional de las entrevistas en profundidad}

\begin{tabular}{|c|c|c|}
\hline Agencias & Anunciantes & Medios digitales \\
\hline $\begin{array}{l}\text { E1. Directora creativa en agencia de } \\
\text { publicidad multinacional. }\end{array}$ & $\begin{array}{l}\text { E1. Director de innovación digital en } \\
\text { empresa energética cotizada. }\end{array}$ & $\begin{array}{l}\text { E1. Jefe de publicidad digital en grupo } \\
\text { multimedia nacional. }\end{array}$ \\
\hline $\begin{array}{l}\text { E2. Head of digital en agencia de } \\
\text { marketing digital nacional. }\end{array}$ & $\begin{array}{l}\text { E2. Brand Manager en empresa de } \\
\text { distribución top } 20 \text { de anunciantes. }\end{array}$ & $\begin{array}{l}\text { E2. Social media manager en medio } \\
\text { especializado en creatividad. }\end{array}$ \\
\hline $\begin{array}{l}\text { E3. Directora de cuentas en agencia } \\
\text { de medios nacional. }\end{array}$ & $\begin{array}{l}\text { E3. Jefe de publicidad en multinacional } \\
\text { de servicios financieros. }\end{array}$ & $\begin{array}{l}\text { E3. Copywriting en departamento } \\
\text { de branded content de periódico } \\
\text { nacional. }\end{array}$ \\
\hline
\end{tabular}

Fuente: elaboración propia.

La muestra final se encuentra dentro del rango de una homogénea, que se da a partir de 6 informantes (Daymon y Halloway, 2011; Guest, Bunce y Johnson, 2006). Estos sujetos entrevistados se constituyen como informantes especiales, al aportar información relevante para el objetivo de la investigación en su condición de expertos, cuestión que permite alcanzar una comprensión teórica fundamentada (Taylor y Bogdan, 1987; Vallés, 2002), al trascender un punto de vista meramente temático (Coffey y Atkinson, 2003). En la novena entrevista se alcanzó el nivel de saturación del discurso en relación con las preguntas semiestructuradas que se habían 
planteado, cuyos conceptos e ideas fueron iterativos, con lo cual se eliminó la posibilidad empírica de que surgieran nuevos discursos relevantes (Glaser y Strauss, 1999; Taylor y Bogdan, 1987).

La estructura de las entrevistas se organizó en tres bloques: perfiles digitales consolidados, perfiles profesionales en los que urge la contratación de personal especializado y perfiles híbridos con mayor previsión de crecimiento. La mayoría de las entrevistas se realizaron en persona, solo una de ellas fue vía telefónica y su duración media fue de 40 minutos. Se codificó el resultado de las respuestas en función de la redundancia de los conceptos identificados en el discurso social (Benavides, 2005), de modo que el contenido adquiriera significación dentro del conjunto en el que se inserta, priorizando el colectivo sobre la persona (Luhmann, 1990). Para tal propósito, en este estudio se consideró el software Nvivo 12 Pro, para el análisis tanto de los datos del discurso empleado por los profesionales del sector publicitario en las entrevistas como de las empresas en las ofertas de trabajo (las palabras frecuentes, su significado y el nivel de representación en la información).

\section{Perfiles para profesionales de la publicidad}

Después de que la Agencia Nacional de Evaluación de la Calidad y Acreditación (Aneca, 2005) publicó el Libro blanco: títulos de grado en comunicación, algunas investigaciones han contemplado los cambios que se han presentado en diversas áreas de trabajo para los profesionales de la publicidad. Categorizamos en la Tabla 2 algunos de esos estudios basándonos en los perfiles delimitados en el Libro blanco para el diseño de las titulaciones en Economía Digital (MIET, 2015), donde el elemento digital es común e indispensable para cualquier función en el mercado laboral europeo. En estas investigaciones destacan figuras que no estaban contempladas en las estructuras clásicas de las empresas, así como roles tradicionales obligados a adaptarse a la situación competitiva del momento, teniendo que mutar responsabilidades y requerimientos de formación en dirección a la tecnología. 


\section{Tabla 2. Perfiles profesionales en publicidad contemplados en investigaciones}

\begin{tabular}{|c|c|}
\hline Área del perfil & Perfil según estudio \\
\hline Diseño web y publicitario & $\begin{array}{l}\text { - Diseñador Front/End (Corredor, 2011; Corredor y Farfán, 2010). } \\
\text { - Diseñador web (Perlado, 2013). } \\
\text { - Director de arte digital (Perlado y Rubio-Romero, 2015; Rooter, 2012). }\end{array}$ \\
\hline $\begin{array}{l}\text { Diseño multimedia, } \\
\text { medios audiovisuales y } \\
\text { new media }\end{array}$ & - Especialista en técnicas audiovisuales (Perlado, 2013; Perlado y Rubio-Romero, 2015). \\
\hline $\begin{array}{l}\text { Creación y gestión de } \\
\text { contenidos digitales }\end{array}$ & $\begin{array}{l}\text { - Desarrollador de contenidos digitales asociados a la marca (Corredor, 2011; Corredor y Farfán, } \\
\text { 2010; Correyero y Balandron, 2010; Rooter, 2012; Núñez, García y Abuín, 2013; Ontsi, 2013; } \\
\text { Vinader, Abuín y García, 2011). }\end{array}$ \\
\hline Social media & $\begin{array}{l}\text { - Community manager o social media manager (Aerco, 2009; Castelló, 2012; Corredor, 2011; } \\
\text { Corredor y Farfán, 2010; Correyero y Balandron, 2010; Infojobs, 2011; Núñez et al., 2013; } \\
\text { Rooter, 2012; Sánchez y Méndez, 2013; Silva, 2016; Vinader et al., 2011). } \\
\text { - Social media analyst (Vinader et al., 2011). } \\
\text { - Online reputation management ORM (Vivar, 2011). }\end{array}$ \\
\hline $\begin{array}{l}\text { Administración y } \\
\text { dirección de empresas } \\
\text { digitales }\end{array}$ & $\begin{array}{l}\text { - Especialista en e-Commerce (Perlado, 2013). } \\
\text { - Big data reader (Perlado y Rubio, 2015). }\end{array}$ \\
\hline Marketing digital & $\begin{array}{l}\text { - Director o especialista de marketing online (Infojobs, 2011; Ontsi, 2013; Perlado, 2013; Rooter, } \\
\text { 2012). } \\
\text { - Responsable de marketing móvil (Corredor, 2011; Corredor y Farfán, 2010). }\end{array}$ \\
\hline Agencia digital & $\begin{array}{l}\text { - Profesional de cuentas experto en nuevos medios (Corredor, 2011; Corredor y Farfán, 2010). } \\
\text { - Planificador de medios experto en nuevos medios (Corredor, 2011; Corredor y Farfán, 2010; } \\
\text { Ontsi, 2013). } \\
\text { - Planificador de medios sociales (Perlado, 2013; Sánchez y Méndez, 2013). } \\
\text { - Responsable SEO (Corredor, 2011; Correyero y Balandron, 2010; Infojobs, 2011; Rooter, 2012; } \\
\text { Sánchez y Méndez, 2013; Vinader et al., 2011; Vivar, 2011). } \\
\text { - Responsable SEM (Corredor, 2011; Correyero y Balandron, 2010; Infojobs, 2011; Perlado, } \\
\text { 2013; Sánchez y Méndez, 2013). } \\
\text { - Chief Blogging officer CBO (Vinader et al., 2011). }\end{array}$ \\
\hline $\begin{array}{l}\text { No contempladas en el } \\
\text { Libro blanco (MIET, 2015) }\end{array}$ & $\begin{array}{l}\text { - User experience (UX) creative (Perlado y Rubio, 2015; Toledano et al., 2017). } \\
\text { - Creative innovation (Perlado y Rubio, 2015; Toledano et al., 2017). } \\
\text { - Creative technologist (Perlado y Rubio, 2015; Toledano et al., 2017; De-Salas et al., 2019). } \\
\text { - Creativo web; redactor social media; redactor ecommerce; creativo SEO/SEM; diseñador } \\
\text { social media; social RR.PP.*; Social commerce; social marketing; social advertising; Marketing y } \\
\text { comunicación (Álvarez et al., 2018). } \\
\text { - User experience designer; technical director; content strategist; connections planner (De-Salas et al., } \\
\text { 2019). }\end{array}$ \\
\hline
\end{tabular}

\footnotetext{
* Relaciones Públicas (RR.PP.).
}

Fuente: elaboración propia a partir de análisis de investigaciones previas. 
Adicional a los perfiles contemplados en áreas del Libro blanco, se localizaron estudios que apuntan a la aparición de perfiles que aúnan dos o más requerimientos. Estas combinaciones resaltan la multidisciplinariedad de forma más marcada, por las competencias solicitadas como parte del impacto digital.

Se tomó como base la información de los estudios y los datos extraídos en la primera etapa de la investigación realizada sobre ofertas de trabajo, para focalizar la interrelación entre las competencias solicitadas (un conocimiento integral de diferentes disciplinas digitales en canales propios, ganados y pagados: web, social, $\mathrm{SEO}+\mathrm{SEM}$, etc.) y las funciones más significativas de los perfiles condicionados a puestos de trabajo, principalmente de aquellos que combinan facetas creativas y técnicas (híbridos). Este primer análisis fue presentado a los entrevistados. Se destaca que en la descripción de $18 \%$ de ofertas de trabajo para profesionales de la publicidad, localizadas en el primer período de análisis, se implicaban competencias de dos o más perfiles, aunque el título de la oferta solo especificaba uno de ellos, predominando ofertas orientadas a la comunicación digital a través de medios sociales.

Ante la información presentada, los entrevistados confirmaron que la industria publicitaria requiere en muchos casos personal con habilidades tecnológicas y publicitarias que implican más de un área específica. Todos los entrevistados coincidieron en destacar perfiles que aúnan el área de social media (principalmente al community manager) con otros, como el de redactor o de diseñador. Se detectó que las empresas llevan a cabo esta tarea para optimizar esfuerzos y atraer a los consumidores de manera efectiva en redes sociales (Gavilanes, Flatten y Brettel, 2018).

De acuerdo con las competencias requeridas, el grupo de las agencias reportó de forma unánime que consideraba más urgente contratar personal especializado y orientado a los medios sociales, por lo que contempló factible para el desarrollo de las funciones, entre las opciones híbridas mostradas, los perfiles de redactor social media, diseñador social media, social advertising y creativo web o en creative innovation. En el grupo de anun- 
ciantes obtuvieron resultados destacados los perfiles híbridos de redactor social media, creativo SEO/SEM y diseñador social media. El grupo de entrevistados pertenecientes a los medios digitales coincidió en apuntar los perfiles de redactor social media, creativo SEO/SEM, diseñador social media y Social RR.PP.

Cuando se les solicitó a los entrevistados que indicaran en cuáles de los tres perfiles profesionales híbridos de respuesta libre, ya fueran generales o específicos, existía previsión en el sector de contratar a mayor número de personas en los próximos años, estos coincidieron en figuras como creativo web (diseñador UX user experience/UI - user interaction) y aquellas que involucran al community manager, destacándose además la figura del redactor, en un perfil más amplio y general que los diferenciados en las categorías profesionales analizadas en los estudios previos. Se observan, aunque con menos mención, figuras híbridas que contemplan funciones $S E O$, social media paid, entre otras.

Al explorar en una segunda etapa los requerimientos de las ofertas de trabajo, se observó un aumento de ofertas que implicaban competencias de dos o más perfiles, vislumbrándose la tendencia prevista por los entrevistados. Los titulares de algunas de ellas (ver Tabla 3) destacaron claramente que se trata de un perfil mixto donde se combina la estrategia y la acción; así que no solo se deduce el efecto híbrido a partir de la descripción de las competencias requeridas. Sumado a estas, otras ofertas de trabajo continúan solicitando un perfil específico, que aúna características de dos o más perfiles.

\section{Tabla 3. Títulos de ofertas de trabajo con un criterio híbrido}

\begin{tabular}{|l|l|}
\hline \multicolumn{1}{|c|}{ Perfil híbrido detectado } & \multicolumn{1}{c|}{ Título de la oferta de trabajo } \\
\hline Redactor web aunado a social media & T1. Social Media + Content Manager \\
& T2. Social Media Design y Content Creator \\
& T3. Copy Creativo / Community Manager \\
& T4. Brand Content / Community Manager \\
& T5. Digital Expert y Community Manager \\
& T6. Social Media Manager and Copy Creative \\
& T7. Community Manager / Copy \\
& T8. Redactor y Community Manager \\
& T9. Content Writer y Social Media Manager \\
\hline
\end{tabular}




\begin{tabular}{|l|l|}
\hline \multicolumn{1}{|c|}{ Perfil híbrido detectado } & \multicolumn{1}{c|}{ Título de la oferta de trabajo } \\
\hline Diseñador web aunado a social media & $\begin{array}{l}\text { T1. Digital + Social Media Manager } \\
\text { T2. Diseño y Redes Sociales }\end{array}$ \\
\hline Creativo aunado al diseñador (UX/UI) & $\begin{array}{l}\text { T1. UX/UI Creative Content Developer } \\
\text { T2. Diseñador Web / Copy } \\
\\
\end{array}$ T3. Dupla Arte y Copy \\
& T4. Dupla Creativa (Copy +Arte) \\
\hline
\end{tabular}

Fuente: elaboración propia a partir del segundo análisis de ofertas de trabajo.

A finales de 2019, también se observaron titulares híbridos en las ofertas, como: content y community manager, community manager/redactor, digital copywriter/UX writer, community/content, copy/social media manager. Se reafirmó la tendencia híbrida, donde el gestor de redes sociales sigue siendo el foco de muchas de esas ofertas.

\section{Perfiles híbridos: aspectos más relevantes}

Aunque existen diferentes figuras en las que un egresado está orientado a desarrollarse profesionalmente, con los conceptos más significativos sobre habilidades y competencias del análisis de contenido de las ofertas de trabajo identificadas en las dos primeras fases y de lo que expresaron los entrevistados, se procedió a modelar las funciones más relevantes para tres perfiles híbridos que, según se detecta, van orientados con más fuerza a las necesidades del mercado laboral publicitario: 1) redactor web aunado a social media, 2) diseñador web aunado a social media y 3 ) creativo aunado al diseñador.

\section{Redactor web aunado a social media}

El perfil requiere capacidades propias de un redactor web de contenidos y gestor de contenidos adaptados al conocimiento de un gestor de redes sociales -comunnity manager-. Se define como un profesional que crea y redacta contenidos para una campaña, producto o marca, estructurándolos en diferentes medios digitales y sociales. Entre sus facultades se incluye velar por la calidad de los contenidos digitales y crear titulares, posts, llamadas de acción, entradas en blogs, noticias, artículos de relevancia, entrevistas, apartados web, de contenido exclusivo y en cualquier otro formato adaptado a medios sociales corporativos y App. En él se destacan además atribuciones como la planificación y operación de la producción y distribución 
de los contenidos de marca y marketing nacionales e internacionales, así como la dinamización y procesamiento de la interrelación con los clientes en pos de resultados y metas. Este perfil debe identificar tendencias e insights en redes sociales para conseguir crear y hacer crecer una comunidad de audiencia alrededor de la marca, estableciendo relaciones y vinculaciones efectivas con dicha comunidad (Facebook, Instagram, Twitter y Linkedln). Por ello deberá crear contenido centrándose en la audiencia con la que interactúa y generar vinculación con la empresa.

\section{Diseñador web aunado a social media}

Un profesional versátil con capacidad de respuesta para coordinar en medios sociales la publicación de contenidos en perfiles corporativos y generar materiales para vincular comunidades. Se requiere un director de arte/diseñador web aunado al community manager que producirá contenidos gráficos para una óptima presencia de una marca en un entorno digital y el impulso de campañas publicitarias, tanto en la web de la empresa como en perfiles sociales corporativos, ilustrando en ellos materiales para la activación de campañas de social media paid. Los contenidos a producir deben permitir la construcción de vínculos a través de conversaciones e iniciativas con la comunidad corporativa, clientes existentes y nuevos clientes, direccionando la dinámica e interacción de comunidades digitales a favor de la empresa.

\section{Creativo aunado al diseñador (UX/UI)}

El perfil conjuga capacidades de los tres perfiles de redactor de contenidos (copywriter), director de arte y diseñador web, para la creación de ideas en un ecosistema digital. Debe desempeñar autónomamente desde la conceptualización y visualización de la creatividad publicitaria hasta su articulación e integración en cualquier medio digital y social, por lo que ha de ser un creador de contenidos especializado en la experiencia de usuario digital. A este profesional se le pide que ejerza funciones que contemplan tanto la idea como la preproducción y/o producción de piezas publicitarias para campañas en el medio digital, desde conceptos, titulares y bodycopys hasta visuales, iconos y layouts adaptables a formatos requeridos, incluyendo la creación de soportes interactivos. Quienes llenen el perfil deben ser profe- 
sionales con conocimientos profundos en publicidad online (diseño digital completo y plena comprensión de medios sociales son esenciales) para implementar dinámicas de contacto tipo user experience (UX) y user interaction (UI) centradas en el público del medio correspondiente, de tal forma que permitan, a través de los contenidos de gamificación, aumentar en este medio el porcentaje de audiencia.

\section{Discusión y conclusiones}

Los cambios económicos han puesto en valor la innovación y oportunidades que exhibe la comunicación digital para crear empleo. Esta evolución ha ido paralela al despliegue del mercado publicitario y de la comunicación de este sector hacia otras formas comunicativas que apuestan por la tecnología. Conjuntamente con informes ofrecidos a nivel general sobre empleabilidad en el sector digital, es preciso extraer y puntualizar en cada uno de los sectores el reto a la demanda prospectiva de figuras profesionales híbridas que exige el mercado laboral en un entorno cada vez más multicanal.

Los perfiles híbridos se han configurado en todos los ámbitos de la comunicación y cada vez son más difusas las líneas que separan la publicidad y las relaciones públicas de la comunicación audiovisual, el periodismo o el marketing. Muestra de ello, por ejemplo, es que la prensa digital ha integrado a periodistas con programadores en los procesos tecnológicos (Marcos, Edo y Parra, 2018). Se observa que la expansión de redes digitales y la evolución de los medios de comunicación han planteado una inclinación más multidisciplinaria en el mercado publicitario para adaptarse a ellos. Este escenario obliga a una redefinición de conocimientos, capacitaciones y perfiles profesionales más tecnológicos que demanda la industria publicitaria en un contexto eminentemente digital. En este sentido es claro que la universidad debe tener en cuenta una formación más especializada para cubrir las necesidades de competencias que exige la publicidad en internet; reformar la oferta formativa y sus currículos educativos con base en esas demandas, para consecuentemente ayudar a los jóvenes en su inserción laboral. Estos cambios en los perfiles profesionales híbridos, versátiles y polivalentes requieren la integración de materias tecnológicas en los planes de estudio, incluyendo las carreras universitarias no técnicas (Vivar 
y Mañas, 2020). La investigación realizada pone de manifiesto que los perfiles profesionales en el ámbito de la comunicación, que tradicionalmente se asociaban de manera explícita a las carreras universitarias de Periodismo, Publicidad y Relaciones Públicas o Comunicación Audiovisual, presentan un espacio de confluencia en el que la palabra comunicación integra a profesionales que provienen de estos diferentes ámbitos y que incluyen habilidades y competencias provenientes del marketing y el desarrollo informático. Este proceso de hibridación se acentuará con la reformulación del mercado de trabajo que ya están provocando la big data, la robótica y la inteligencia artificial (Nam, 2019).

Con esas premisas, en este artículo se identificó y describió la percepción sobre algunos perfiles híbridos en los que existe previsión de contratar a mayor número de personas en los próximos años para desempeñar puestos de trabajo en el sector publicitario. La investigación extrajo las funciones generales de perfiles híbridos y transversales que, cimentados en un conocimiento holístico de la disciplina, llegarían a abordar capacidades concretas que reflejen no solo una capacidad creativa sino una inteligencia analítica y consultiva para combinarlas estratégicamente con las tecnologías. Se ve cómo continúa la evolución de perfiles profesionales al ritmo y relevancia que estas tienen en las labores económicas. Se perfila un eje de acción más amplio en varias áreas de desempeño y sectores laborales en los que antes no se vislumbraba a este profesional de la publicidad y donde cada vez funciona más la polivalencia profesional.

Se requiere la presencia de un publicitario más multidisciplinario y tecnológico que permita materializar y compartir más eficientemente las ideas con los consumidores, donde el gestor de redes sociales y comunidades digitales se manifiesta como el epicentro de las diversas áreas solapadas en términos de habilidades y que reclaman los empleadores para una comunicación crecidamente interactiva con su audiencia en internet. En el horizonte de la publicidad en España -al igual que lo hicieron Corredor y Farfán (2010), Silva (2016) y se refleja en el informe sobre el futuro del trabajo en España (Adecco, 2016) - se muestra la presencia con más ímpetu del community manager como una de las profesiones más destacadas y 
consolidadas, fundamental en el panorama de la comunicación corporativa, pero haciendo notar que el sector apuesta por un perfil aún más amplio ygeneral que aúna competencias de otros perfiles, ante las necesidades que se les presentan para adaptarse a los distintos contextos.

Son numerosos los autores que han puesto de manifiesto que las profesiones relacionadas con las redes sociales exigen cada vez más requisitos en un contexto de disminución salarial (Duffy y Schwartz, 2018; Silva, 2016), siendo los perfiles mejor remunerados aquellos que incluyen el desarrollo de aplicaciones, la gestión de datos y la experiencia de usuario (Jiménez y Mañas, 2018). Esto ha provocado una tendencia a la hibridación, que se observa mayoritariamente en el perfil profesional de community manager, que evoluciona hacia la capacidad de generar contenidos $y$ otras funciones más próximas al marketing digital y la gestión de influencers (Mañas y Jiménez, 2019).

El análisis ha permitido configurar preferencias de la fuerza laboral por perfiles híbridos como el de redactor o el de diseñador asociado a los social media y deja ver la interrelación de habilidades entre las figuras publicitarias. Se plantean y se desglosan funciones de perfiles profesionales cuyas diferenciaciones conciernen en parte al dominio de habilidades tecnológicas. Esto refuerza lo expuesto por Perlado y Rubio (2015), en el sentido de que se tienen más en cuenta las habilidades concretas que deben asumirse para un puesto determinado, en lugar de la categorización en sí del perfil ya prestablecido, allí donde las responsabilidades a cubrir requieren perfiles profesionales con roles más completos y dinámicos, donde el "saber hacer" (competencias) es mucho más imperativo para cualquier puesto de trabajo. Por tanto, en el tránsito de los profesionales demandados hoy en día, en términos de conocimientos y capacitaciones, desde el "saber" hacia el "saber hacer", es necesario una formación universitaria y profesional más híbrida que mejore índices y tiempos de inserción en el mercado.

Este trabajo pretende contribuir a las llamadas que se han realizado desde la Unión Europea a la mejora y atractivo de las carreras universitarias -Gran Coalición para el Empleo Digital (European Commission, 2013)-, 
a través de la formación de las cualificaciones exigidas para el empleo y, con ello, a reducir la brecha entre oferta y demanda. Los programas educativos deben ser dotados de una gran flexibilidad para esta adaptación, donde es necesaria una visión integradora de agentes sociales que permitan capitalizar todas las oportunidades.

\section{Referencias}

Adecco (2016). Informe Adecco sobre el futuro del trabajo en España. Madrid: Adecco. Recuperado de: https://www.ceu.es/joblab/documentacion/informeAdecco.pdf

Aerco - Asociación Española de Responsables de Comunidades OnLine (2009). La función del community manager. Cómo las empresas están organizándose para crear y hacer crecer sus comunidades. Aerco, Territorio Creativo. Recuperado de: http://www.maestrosdelweb. com/images/2010/04/community-manager.pdf

Álvarez-Flores, E. P., Núñez-Gómez, P. y Olivares-Santamarina, J.P. (2018). Perfiles profesionales y salidas laborales para graduados en Publicidad y Relaciones Públicas: de la especialización a la hibridación. El Profesional de la Información, 27(1), 136-147. DOI: https://doi. org/10.3145/epi.2018.ene.13

Aneca - Agencia Nacional de Evaluación de la Calidad y Acreditación (2005). Libro Blanco. Títulos de Grado en Comunicación. Aneca. Recuperado de: http://www.aneca.es/var/media/150336/libroblanco_comunicacion_def.pdf

Armendáriz, E. (2015). El nuevo perfil del profesional de la Comunicación y las Relaciones Públicas. Una visión desde la perspectiva del mercado. Revista Internacional de Relaciones Públicas, 5(9), 151-178. DOI: http://dx.doi.org/10.5783/RIRP-9-2015-09-153-178 
Benavides Delgado, J. (2005). Nuevas propuestas para el análisis del lenguaje en los medios. Questiones Publicitarias, 1(10), 13-33. DOI: https://doi.org/10.5565/rev/qp.154

Besalú-Casademont, R., Schena, J. y Sánchez-Sánchez, C. (2017). Competencias más relevantes en los estudios de Comunicación Audiovisual. Las percepciones de profesionales, docentes y estudiantes. Revista Latina de Comunicación Social, 72, 1536-1553. DOI: http:// doi.org/10.4185/RLCS-2017-1233

Bravo, J. y Madinaveitia, E. (2015). Introducción: La Publicidad en 2014: Transformaciones ante el reto digital. Telos, 99, 56-63. Recuperado de: https://dialnet.unirioja.es/servlet/articulo?codigo $=4955643$

Castelló Martínez, A. (2012). El estudio de la comunicación publicitaria online en el Grado en Publicidad y RR.PP. Questiones Publicitarias, 1(17), 1-23. Recuperado de: https://rua.ua.es/dspace/ bitstream/10045/34463/1/2012_Castello_QuestionesPublicitarias.pdf

Coffey, A. y Atkinson, P. (2003). Encontrar el sentido a los datos cualitativos. Estrategias complementarias de investigación. Medellín: Contus.

Corredor Lanas, P. (2011). En clave digital. Nuevos profesionales en publicidad. Telos: Cuadernos de Comunicación e Innovación, 87, 97-100. Recuperado de: https://dialnet.unirioja.es/servlet/ articulo? codigo $=3656826$

Corredor Lanas, P. y Farfán Montero, J. (2010). Demandas y formación: Nuevos perfiles profesionales para la publicidad en España. Pensar la Publicidad: Revista Internacional de Investigaciones Publicitarias, 4(1), 97-116. Recuperado de: https://revistas.ucm.es/index. php/PEPU/article/view/PEPU1010120097A

Correyero Ruíz, B. y Baladrón Pazos, A. J. (2010). Nuevos perfiles profesionales en el entorno digital: un desafío para la formación de comuni- 
cadores desde el EEES. España: Universidad de la Laguna. Actas II Congreso Internacional Latina de Comunicación Social. Recuperado de: http://www.revistalatinacs.org/10SLCS/actas_2010/044_ Correyero.pdf

Daymon, C. y Holloway, I. (2011). Qualitative research methods in public relations and marketing communications. Nueva York: Routledge.

De-Salas-Nestares, I., Martínez-Sáez,J.y Roca-Hueso, V. (2019). Los nuevos perfiles profesionales como elemento transformador de la industria publicitaria. Revista Mediterránea de Comunicación, 10(2), 11-28. DOI: https://www.doi.org/10.14198/MEDCOM2019.10.2.16

Duffy, B. E. y Schwartz, B. (2018). Digital 'women's work?’: Job recruitment ads and the feminization of social media employment. New Media y Society, 20(8), 2972-2989. DOI: https://doi. org/10.1177/1461444817738237

European Commission (2013). Gran coalición para la creación de empleos en la economía digital. European Commission. Recuperado de: http:// europa.eu/rapid/press-release_IP-13-182_es.htm

Fuller, M., Heijne-Penninga, M., Kamans, E., Van Vuuren, M., De Jong, M. y Wolfensberger, M. (2018). Identifying competence characteristics for excellent communication professionals: A work field perspective. Journal of Communication Management, 22(2), 233-252. DOI: https://doi.org/10.1108/JCOM-07-2016-0051

Gavilanes, J. M., Flatten, T. Ch. y Brettel, M. (2018). Content strategies for digital consumer engagement in social networks: Why advertising is an antecedent of engagement. Journal of Advertising, 47(1), 4-23. DOI: https://doi.org/10.1080/00913367.2017.1405751

Glaser, B. G. y Strauss, A. L. (1999). The discovery of grounded theory. Strategies for qualitative research. Piscataway: Aldine Transaction. 
Guest, G., Bunce, A. yJohnson, L. (2006). How many interviews are enough?: An experiment with data saturation and variability. Field Methods, 18(1), 59-82.DOI: https://doi.org/10.1177/1525822X05279903

IAB (2011). Observatorio del mercado laboral de los profesionales del marketing, la comunicación y la publicidad digital, 2a Oleada. IAB España. Recuperado de: https://es.slideshare.net/IAB_Spain/observatorio-del-mercado-laboral-de-los-profesionales-del-marketing-la-comunicacin-y-la-publicidad-digital

Infojobs (2011). Profesiones relacionadas con internet en el mercado laboral español 2010. Asociación Española de la Economía Digital. Recuperado de: https://www.adigital.org/informes-estudios/ profesiones-relacionadas-con-internet-en-el-mercado-laboral-espanol-2010/

Jiménez-Gómez, I. y Mañas-Viniegra, L. (2018). Ofertas de empleo mejor remuneradas en comunicación y diseño: nuevos perfiles y efecto full-stack. Doxa Comunicación, 27, 239-251. DOI: https://doi. org/10.31921/doxacom.n27a12

Kumar, V. y Gupta, S. (2016). Conceptualizing the evolution and future of advertising. Journal of Advertising, 45(3), 302-317. DOI: http:// doi.org/10.1080/00913367.2016.1199335

Kvale, S. (2011). Las entrevistas en investigación cualitativa. Madrid: Morata.

López-Berna, S., Papí-Gálvez, N. y Martín, M. (2016). La revisión de los grados universitarios: valoración de la convergencia europea del Grado en Publicidad y Relaciones Públicas en España. Doxa Comunicación, 23, 47-71. DOI: https://doi.org/10.31921/doxacom.n23a2

Luhmann, N. (1990). Sociedad y sistema: la ambición de la teoría. Barcelona: Paidós/ICE-UAB. 
Malhotra, N., Hall, J., Shaw, M. y Oppenheim, P. (2002). Marketing research. An applied orientation (2 ed.). Englewood Cliffs: Prentice-Hall.

Mañas-Viniegra, L. y Jiménez-Gómez, I. (2019). Evolución del perfil profesional del community manager durante la década 2009-2018. El Profesional de la Información, 28(4), e280403. DOI: https://doi. org/10.3145/epi.2019.jul.03

Marcos-Recio, J. C., Edo-Bolós, C. y Parra-Valcarce, D. (2018). Remaining challenges for digital newspapers regarding informative updates: case studies in the Spanish media. Communication y Society, 31(2), 5169. Recuperado de: https://dadun.unav.edu/handle/10171/55710

McEnnis, S. (2017). Playing on the same pitch. Attitudes of sports journalists towards fan blogger. Digital Journalism, 5(5), 549-566. DOI: https://doi.org/10.1080/21670811.2016.1246374

MIET - Ministerio de Industria, Energía y Turismo (2015). Libro blanco para el diseño de las titulaciones universitarias en el marco de la economía digital. Madrid: Agenda Digital para España, MIET. Recuperado de: http://www.ccii.es/images/ccii/recursos/Libro-Blanco.pdf

Nam, T. (2019). Technology usage, expected job sustainability, and perceived job insecurity. Technological Forecasting and Social Change, 138, 155-165. DOI: https://doi.org/10.1016/j.techfore.2018.08.017

Núñez, P., García, A. y Abuín, N. (2013). Profesionales digitales en publicidad y comunicación. Una aproximación a las necesidades del mercado laboral. Cuadernos de Información y Comunicación, 18, 177-187. DOI: http://dx.doi.org/10.5209/rev_CIYC.2013. v18.41723

Observatorio de la Publicidad en España (2015). La comunicación comercial en cambio permanente. Asociación Española de Anunciantes. Recuperado de: https://www.researchgate.net/profile/ Elena-Fernandez-Blanco/publication/307607893_Observato- 
rio_de_la_Publicidad_2015_La_comunicacion_comercial en_cambio_permanente/links/57cd66b208ae3ac722b84a31/ Observatorio-de-la-Publicidad-2015-La-comunicacion-comercial-en-cambio-permanente.pdf

Ontsi - Observatorio Nacional de las Telecomunicaciones y de la Sociedad de la Información (2013). Oferta y demanda de profesionales en contenidos digitales. España: Ministerio de Asuntos Económicos y Transformación Digital. Recuperado de: https://www.ontsi.red. es/sites/ontsi/files/informe_oferta_y_demanda_de_profesionales_en_contenidos_digitales.pdf

Ontsi - Observatorio Nacional de las Telecomunicaciones y de la Sociedad de la Información (2017). Informe anual del sector de los contenidos digitales en España 2016. España: Ministerio de Energía, Turismo y Agenda Digital. Recuperado de: https://www.ontsi.red. es/sites/ontsi/files/Informe\%20Sector\%20de\%20los\%20Contenidos\%20Digitales\%202016.pdf

Perlado Lamo de Espinosa, M. (2013). Nuevas oportunidades en la comunicación digital: nuevos perfiles y competencias. Prospectivas y Tendencias para la Comunicación en el Siglo XXI, 17(6), 429-440. Recuperado de: https://dialnet.unirioja.es/servlet/ articulo? codigo $=4582521$

Perlado Lamo de Espinosa, M. y Rubio-Romero, J. (2015). Competencias, habilidades y formación del creativo publicitario en la era digital. Creatividad y Sociedad, 23, 6-34. Recuperado de: https://dialnet. unirioja.es/servlet/articulo? codigo $=5552945$

Perlado-Lamo de Espinoza, M., Saavedra, M. y Rubio-Romero, J. (2016). La formación universitaria en España dentro del ámbito de la comunicación corporativa. En M. Perlado Lamo de Espinosa y C. Cachan Alcolea (eds.), Competencias y perfiles profesionales en el ámbito de la comunicación (pp. 263-274). Madrid: Dykinson. 
Perlado-Lamo-de-Espinosa, M., Papí-Gálvez, N. y Bergaz-Portolés, M. (2019). Del planificador de medios al experto en medios: el efecto digital en la publicidad. Comunicar, XXVII(59), 105-114. DOI: https://doi.org/10.3916/C59-2019-10

Rooter (2012). Perfiles profesionales más demandados en el ámbito de los contenidos digitales en España 2012-2017. Madrid: Fundación Tecnológica de la Información. Recuperado de: http://ametic.es/ sites/default/files/pafet_vii_perfiles_profesionales_cd_fti-rooter_1.pdf

Sánchez González, H. y Méndez Muros, S. (2013). ¿Perfiles profesionales 2.0? Una aproximación a la correlación entre la demanda laboral y la formación universitaria. Estudios sobre el Mensaje Periodístico, 19, 981-993. DOI: http://dx.doi.org/10.5209/rev_ESMP.2013. v19.42183

Sánchez-Sánchez, C. y Fernández-Cavia, J. (2018). Percepción de profesionales y académicos sobre los conocimientos y competencias necesarios en el publicitario actual. Revista Latina de Comunicación Social, 73, 228-263. DOI: https://doi.org/10.4185/RLCS2018-1254

Schultz, D. (2016). The future of advertising or whatever we're going to call it. Journal of Advertising, 45(3), 276-285. DOI: http://doi.or $\mathrm{g} / 10.1080 / 00913367.2016 .1185061$

Silva-Robles, C. (2016). Perfil del Community Manager en las agencias de publicidad y relaciones públicas de España. El Profesional de la Información, 25(2), 237-245. DOI: https://doi.org/10.3145/ epi.2016.mar.10

Taylor, S. J. y Bogdan, R. (1987). Introducción a los métodos cualitativos de investigación. Barcelona: Paidós. 
Toledano, F., Miguel, B. y Grijalba, N. (2017). La transformación de la industria publicitaria: nuevos perfiles y competencias del creativo publicitario. En M. Perlado Lamo de Espinosa y C. Cachán Alcolea (eds.), Competencias y perfiles profesionales en el ámbito de la comunicación (pp. 55-66). Madrid: Dykinson.

Vallés, M. (2002). Entrevistas cualitativas. Cuadernos metodológicos. Madrid: CIS.

Ventura, R., Roca-Cuberes, C. y Corral-Rodríguez, A. (2018). Comunicación digital interactiva: valoración de profesionales, docentes y estudiantes del área de la comunicación sobre las competencias académicas y los perfiles profesionales. Revista Latina de Comunicación Social, 73, 331-351. DOI: https://doi.org/10.4185/ RLCS-2018-1258

Vilches, L. (coord.) (2011). La investigación en comunicación: métodos y técnicas en la era digital. Barcelona: Gedisa.

Vinader Segura, R., Abuín Vences, N. y García García, A. (2011). El impacto de la web 2.0 en los perfiles profesionales del sector audiovisual. Telos: Cuadernos de Comunicación e Innovación, 87, 1-11. https://telos.fundaciontelefonica.com/archivo/numero087/el-impacto-dela-web-2-0-en-los-perfiles-profesionales-del-sector-audiovisual/

Vivar Zurita, H. (2011). Nuevos perfiles profesionales para una comunicación digital. Telos: Cuadernos de Comunicación e Innovación, 87, 1-4. https://dialnet.unirioja.es/ejemplar/280316

Vivar-Zurita, H. y Mañas-Viniegra, L. (2020). La sociedad digital y su empleabilidad en las áreas de Información y Comunicación: nuevos retos para la redefinición de las competencias profesionales en los planes de estudio universitarios. En H. Vivar-Zurita (ed.), Sociedad digital y empleabilidad en comunicación (pp. 19-38). Salamanca: Comunicación Social. 